\title{
Kallikrein Inactivator Unit per Milliliter
}

National Cancer Institute

\section{Source}

National Cancer Institute. Kallikrein Inactivator Unit per Milliliter. NCI Thesaurus. Code C73531.

An arbitrary unit of a kallikrein inactivator concentration equal to the concentration at which one milliliter of the mixture contains one unit of the kallikrein inactivator. 